\title{
Faces retain attention
}

\author{
MARKUS BINDEMANN and A. MIKE BURTON \\ University of Glasgow, Glasgow, Scotland \\ IGNACE T. C. HOOGE \\ Utrecht University, Utrecht, The Netherlands \\ ROB JENKINS \\ University of Glasgow, Glasgow, Scotland \\ and \\ EDWARD H. F. DE HAAN \\ Utrecht University, Utrecht, The Netherlands
}

\begin{abstract}
In the present study, we investigated whether faces have an advantage in retaining attention over other stimulus categories. In three experiments, subjects were asked to focus on a central go/no-go signal before classifying a concurrently presented peripheral line target. In Experiment 1, the go/no-go signal could be superimposed on photographs of upright famous faces, matching inverted faces, or meaningful objects. Experiments 2 and 3 tested upright and inverted unfamiliar faces, printed names, and another class of meaningful objects in an identical design. A fourth experiment provided a replication of Experiment 1, but with a 1,000-msec stimulus onset asynchrony between the onset of the central face/nonface stimuli and the peripheral targets. In all the experiments, the presence of an upright face significantly delayed target response times, in comparison with each of the other stimulus categories. These results suggest a general attentional bias, so that it is particularly difficult to disengage processing resources from faces.
\end{abstract}

Faces constitute a class of visual stimuli of immense biological and social importance to us, and there is some evidence that faces may have an advantage over other stimulus categories in capturing visual attention. This evidence includes studies of neuropsychological patients with hemispatial visual neglect (Vuilleumier, 2000) and of neurologically normal subjects (Mack, Pappas, Silverman, \& Gay, 2002; Shelley-Tremblay \& Mack, 1999), demonstrating that faces are reported more often than nonface objects under conditions that should make detection difficult. Furthermore, some authors have reported that subjects are able to detect changes to faces more easily than changes to other objects in flicker paradigm experiments (Ro, Russell, \& Lavie, 2001; although see Palermo \& Rhodes, 2003, for contrary evidence). In the experiments reported here, we addressed a different problem: Do faces retain attention disproportionately, in comparison with other stimuli?

Studies of attention retention have previously emphasized the emotional aspects of the stimuli. For example, Bradley, Mogg, Falla, and Hamilton (1998) used faces to

This work was supported by an ESRC postgraduate studentship (R42200134060) and an EPS Study Visit Grant to M.B. Correspondence concerning this article should be addressed to M. Bindemann, Department of Psychology, University of Glasgow, Glasgow G12 8QQ, Scotland (e-mail: markus@psy.gla.ac.uk). cue the location of a dot probe target to show that there was an attentional bias toward threatening faces, by comparison with happy or neutral faces. However, this bias existed only for subjects with high trait anxiety and was particularly pronounced on invalidly cued trials, as indexed by increased response latencies. Analogous effects have been demonstrated with stimuli other than faces. For example, there appears to be an attentional bias toward threatening pictures (see, e.g., Yiend \& Mathews, 2001) and threatening words (e.g., Amir, Elias, Klumpp, \& Przeworski, 2003; Fox, Russo, Bowles, \& Dutton, 2001), by comparison with nonthreatening counterparts. Collectively, these studies suggest that attentional resources can be tied to a spatial location by some types of stimuli. However, none of these studies addressed whether faces generally are particularly efficient at retaining attention, since faces were never directly compared with other classes of stimuli within the same experiment.

To investigate this possibility, we devised a simple classification task in which subjects focused on a central go/ no-go signal before, on go trials, responding to a peripheral line target. Go trials, designated by a green dot, therefore required an attentional shift from the location of the signal to that of the target. No-go trials, designated by red dots, required only a target-neutral buttonpress to initiate the next trial. The go/no-go signal could be superimposed on a blank background or on to-be-ignored face images and different nonface comparisons. Note that identifying 
the color of the go/no-go signal is a task thought to place minimal demands on attention (see, e.g., Treisman, 1993), which should make it impossible not to process other information presented at fixation (e.g., Lavie, 1995, 2000). If faces have an advantage in retaining attention, target response times (RTs) should thus be slowed more by the presence of a face than by other visual stimuli. This hypothesis was tested over four experiments.

\section{EXPERIMENT 1}

\section{Method}

Twenty volunteer subjects were shown displays containing a central dot indicating go trials (green) or no-go trials (red). The dot measured $0.2^{\circ}$ of visual angle (VA) in diameter at a viewing distance of $60 \mathrm{~cm}$ (held constant by means of a chinrest) and was flanked by a vertical line on one side and a horizontal line on the other. These lines were presented in black at a size of $0.1^{\circ} \times 0.4^{\circ}$ of VA and were positioned $4.6^{\circ}$ to the left and to the right of center. The position of these lines was counterbalanced throughout the experiment, so that each line occurred equally often on the left and the right. In addition to the dot signal and line targets, these displays could contain (1) no further image, (2) an upright famous face, (3) an inverted famous face, or (4) a meaningful nonface object - in this case, an image of a fruit. Photographs of three female celebrities
(Pamela Anderson, Marilyn Monroe, and Britney Spears) and three types of fruits (apple, plum, and grapes) were used for these conditions. These were converted to grayscale, were cropped to remove any extraneous background, and were positioned behind the fixation dot at a size of approximately $2.3^{\circ} \times 2.9^{\circ}$ of VA (for examples, see Figure 1). Note that the inverted face and fruit conditions were intended to serve as controls for the upright face stimuli. Inverted faces perfectly match their upright equivalents in terms of spatial frequency, complexity, and stimulus homogeneity but are perceived and recognized so poorly that it has been suggested that they may be processed as objects, rather than as faces (see, e.g., Farah, Wilson, Drain, \& Tanaka, 1995; Moscovitch, Winocur, \& Behrmann, 1997). In contrast to inverted faces, the fruit stimuli were not equated to the low-level visual properties of the face stimuli but were included to provide a meaningful object comparison.

Each trial began with a black fixation dot $(750 \mathrm{msec})$, followed by a stimulus display $(200 \mathrm{msec})$ and a blank screen until a response was registered. A practice block of 36 blank trials (i.e., with no face/ nonface image present) was followed by four experimental blocks of 36 trials, one for each condition. Trial order was randomized within blocks, but go trials occurred twice as frequently as no-go trials. The experimental conditions were blocked to decrease the potential risk of popout (see Palermo \& Rhodes, 2003), but block order was counterbalanced across subjects across the experiment.

The subjects were requested to focus on the center of the screen at the start of each trial and, if the experimental display contained a green fixation dot (on go trials), to make a speeded judgment regard-

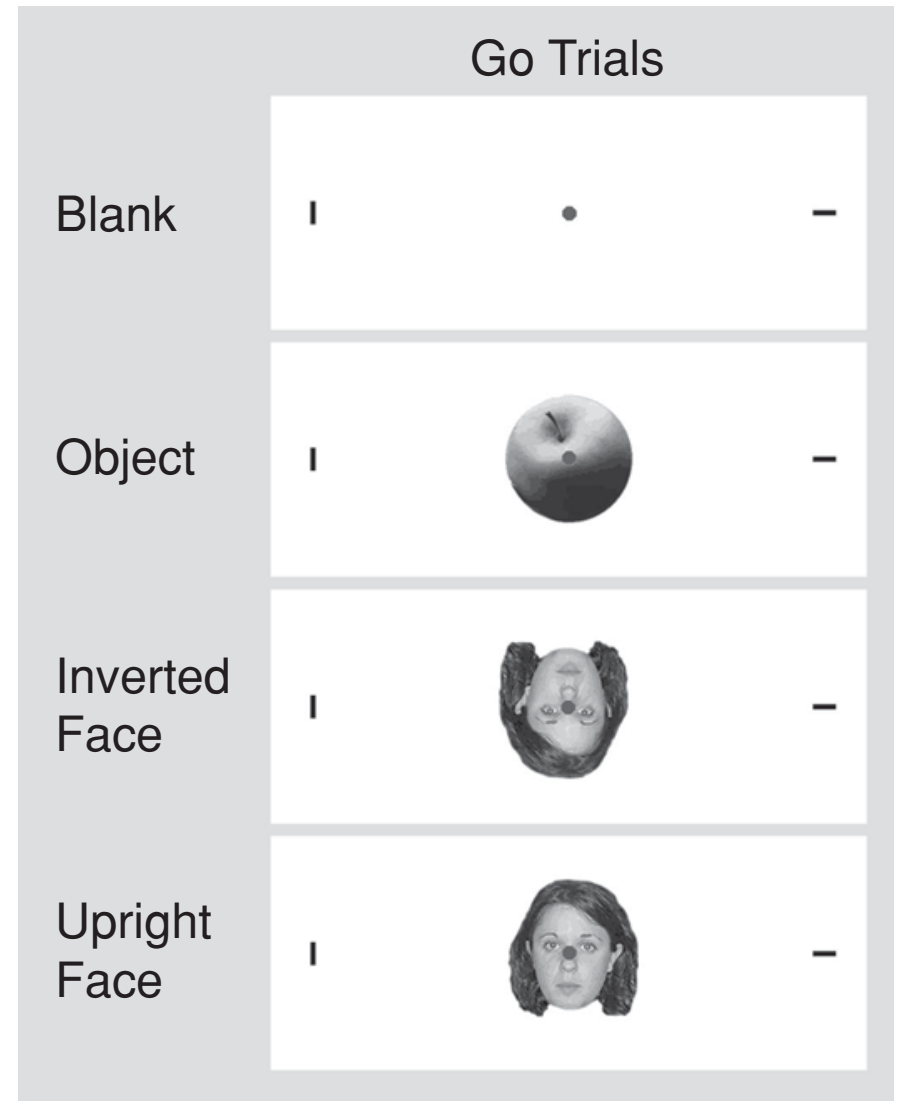

Figure 1. Example displays for Experiments 1 and 2. The central dot probe, here in grayscale, could be green on go trials or red on no-go trials. The vertical line target was equally likely to occur in the left or the right periphery, with the horizontal line always appearing on the opposite side. 
ing the location of the vertical line target (i.e., left or right of fixation) via a speeded two-choice keypress response. For no-go trials, designated by a red fixation dot, the subjects were asked to ignore the line targets and to press the space key to initiate the next trial. In addition, the subjects were emphatically instructed to ignore any other stimuli that might be presented at fixation.

\section{Results}

The data of principal interest were the median correct RTs and error rates for the go conditions. The cross-subject averages of these RTs and error rates are shown in Table 1. A one-way ANOVA of RTs (blank vs. object vs. inverted face vs. upright face) showed an effect of condition $[F(3,57)=$ $16.58, p<.01]$. As is suggested in Table 1 , responses in the blank condition were significantly faster than those for all the other conditions (Tukey HSD test, $p<.01$ ). More important, RTs in the upright face condition were also longer than those in all the other conditions $(p<.05)$. However, no difference between the inverted face and the object conditions was found. Analogous analyses on the error data for the go trials showed no significant effect of condition $[F(3,57)=2.16]$.

To indicate compliance with the full task demands and for completeness, two further one-way ANOVAs were conducted on the RTs and the error rates for the no-go conditions. Table 1 shows that the no-go conditions follow an RT pattern similar to that for the go conditions, with the slowest responses occurring in the upright face condition. However, for the no-go conditions these differences did not reach significance $[F(3,57)=2.01]$. Similarly, no main effect in error rates was found $[F(3,57)=1.20]$.

\section{Discussion}

Experiment 1 demonstrates a clear bias for upright faces, suggesting that it is particularly difficult to shift attention from the location of a face to that of a peripheral target. This effect is seemingly independent of the low-level characteristics of the face images, since similar increases in RTs were not observed for inverted faces. The effect also does not appear to reflect a speed-accuracy trade-off. Indeed, the error pattern indicates that presenting upright faces did not affect whether the line targets were processed (see, e.g., Lavie, 1995) but, rather, affected only the timing of responses to these targets. Note that in line with this reasoning, a similar but nonsignificant response pattern was also obtained for no-go trials, which did not require the processing of the line targets.

Table 1

Mean Reaction Times (RTs, in Milliseconds), Standard Deviations ( $S D s)$, and Percentages of Errors (\%E) as Functions of the Go and No-Go Conditions in Experiment 1

\begin{tabular}{lrrrrrrr}
\hline & \multicolumn{3}{c}{ Go Condition } & & \multicolumn{3}{c}{ No-Go Condition } \\
\cline { 2 - 3 } \cline { 7 - 8 } Stimulus & RT & $S D$ & $\% \mathrm{E}$ & & RT & $S D$ & $\% \mathrm{E}$ \\
\hline Blank & 509 & 93 & 1 & & 560 & 92 & 6 \\
Object & 558 & 83 & 4 & & 570 & 115 & 7 \\
Inverted face & 561 & 95 & 4 & & 579 & 124 & 6 \\
Upright face & 596 & 101 & 3 & & 611 & 112 & 10 \\
\hline
\end{tabular}

\section{EXPERIMENT 2}

Experiment 2 was conducted to examine whether the attention retention bias for upright faces, as measured with photographs of famous faces in Experiment 1, could also be tapped by unfamiliar face stimuli. Some postperceptual mechanisms are available only to familiar face processing, such as person recognition and semantic access (see, e.g., Bruce \& Young, 1986; Burton, Bruce, \& Hancock, 1999; Burton \& Young, 1999; Young \& Burton, 1999). Consequently, the possibility exists that a face bias applies only to higher level processes, rather than reflecting a general bias that can be tapped by any face stimuli. In Experiment 2, the stimuli and procedure were, therefore, kept identical to those in Experiment 1, except that the upright and inverted famous face stimuli were now replaced by photographs of three unfamiliar female models.

\section{Method}

Twenty-eight volunteer subjects were recruited, none of whom had taken part in the previous experiment. The celebrities' faces from Experiment 1 were replaced with equivalently prepared photographs of unfamiliar faces. In all other respects, the design and procedure were identical to those in the previous experiment.

\section{Results}

Table 2 shows the cross-subject averages of the median correct RTs and error rates for the go and the no-go conditions. For go RTs, a one-way ANOVA showed an effect of condition $[F(3,81)=33.16, p<.01]$. As in Experiment 1 , this reflected the fact that the shortest RTs were those in the blank condition (Tukey HSD, $p<.01$ ) and the longest were those in the upright face condition, in comparison with each of the other conditions $(p<.05)$. No difference between the inverted face and the object conditions was found. An analogous analysis of the go error rates also revealed a main effect of condition $[F(3,81)=4.06, p>$ .05 ], reflecting more errors in the object condition than in the blank condition.

For completeness, the no-go data were analyzed in the same way. A one-way ANOVA revealed a main effect of condition for RTs $[F(3,81)=6.97, p<.01]$, reflecting significantly faster responses in the blank condition than in the object and the upright face conditions $(p<.05)$. However, although RTs were also longest in the upright face condition, no other comparisons were significant. A further ANOVA showed no effect in error rates $[F(3,81)=$ $0.50]$.

\section{Comparison of Famous Faces (Experiment 1) and Unfamiliar Faces (Experiment 2)}

To examine any influence of face familiarity, a 2 (familiarity: famous, unfamiliar) $\times 4$ (experimental condition: blank, object, inverted face, upright face) mixed ANOVA was also conducted on the go RTs for Experiments 1 and 2 . This revealed an effect of experimental condition $[F(3,138)=45.78, p<.01]$, reflecting significant differences between all of the within-subjects conditions (Tukey HSD, all $p \mathrm{~s}<.01$ ), except between the object and 
Table 2

Mean Reaction Times (RTs, in Milliseconds), Standard

Deviations (SDs), and Percentages of Errors (\%E) as Functions of the Go and No-Go Conditions in Experiment 2

\begin{tabular}{|c|c|c|c|c|c|c|}
\hline \multirow[b]{2}{*}{ Stimulus } & \multicolumn{3}{|c|}{ Go Condition } & \multicolumn{3}{|c|}{ No-Go Condition } \\
\hline & RT & $S D$ & $\% \mathrm{E}$ & RT & $S D$ & $\% \mathrm{E}$ \\
\hline Blank & 493 & 84 & 4 & 598 & 107 & 10 \\
\hline Object & 570 & 106 & 9 & 653 & 108 & 12 \\
\hline Inverted face & 571 & 103 & 6 & 639 & 106 & 9 \\
\hline Upright face & 609 & 107 & 6 & 672 & 94 & 9 \\
\hline
\end{tabular}

the inverted face conditions. However, there was no main effect of familiarity $[F(1,138)=0.03]$ and no interaction $[F(3,138)=1.21]$.

\section{Discussion}

Experiment 2 replicated the important aspects of Experiment 1 with unfamiliar faces. The longest RTs were observed again when an upright face was presented at fixation, in comparison with a blank background or when an inverted face or a nonface object was presented. As before, a similar nonsignificant RT pattern was also found for the no-go conditions, providing some further support to the notion of an attention retention bias for faces. Moreover, a between-subjects analysis of the go RTs of Experiments 1 and 2 suggests that whether famous or unfamiliar faces are used as stimuli, there may be no difference in this bias. Therefore, these results indicate that the findings of Experiment 1 are not dependent on mechanisms associated with familiar face processing but that attention retention may be a property of faces in general.

\section{EXPERIMENT 3}

Experiment 2 showed that the face bias in Experiment 1 did not depend on the familiarity of the face stimuli. In Experiment 3, we examined whether this bias might have its source in the personhood of face stimuli. Thus, we asked whether people's names might have the same attentionretaining properties as faces. Unlike the pictures of inverted faces that were used in the previous experiments, this manipulation eliminated all surface similarities with the upright faces but gave all the stimuli the same status as persons. Models of person recognition postulate separate visual processing routes for faces and names, which then converge at more abstract representations, known as person identity nodes (Bruce \& Young, 1986; Burton et al., 1999; Burton \& Young, 1999; Young \& Burton, 1999), and here, by analogy, we further examined the level of abstraction at which our attention retention effect for faces occurs. If an attention retention bias reflects the personhood of face stimuli, one might expect a similar bias for names. On the other hand, if any retention bias is specific to faces, faces should outperform names under the present circumstances.

In order to extend the range of stimuli with which to compare faces, we also used a new class of visual stim- uli in this experiment. The fruit pictures used in Experiments 1 and 2 were replaced with photographs of flags.

\section{Method}

Twenty-four new volunteer subjects were recruited. The upright and inverted face displays were those used in Experiment 1-that is, images of three famous women. The name stimuli consisted of the names of these celebrities, printed in black 18-point Arial font, with forenames printed just above fixation and surnames just below. They measured between $1.3^{\circ}$ (the shortest name) and $1.7^{\circ}$ (the longest name) of VA in width. Greek, South African, and Swiss flag stimuli were edited in the same way as the face stimuli in order to provide new object comparisons and were presented in grayscale at a size of $2.3^{\circ} \times 2.9^{\circ}$ of VA.

\section{Results}

The means of the median correct RTs and error rates for the go and no-go conditions are shown in Table 3. A one-way ANOVA showed a main effect of condition for go RTs $[F(3,69)=33.48, p<.01]$, with significantly slower responses in the face condition than in the blank, name, and object conditions (Tukey HSD, $p<.05$ ). As before, RTs were also shorter in the blank condition than in each of the other conditions $(p<.01)$, but the slight difference between the name and the object conditions did not reach significance. An ANOVA of the go error rates also showed an effect of condition $[F(3,69)=4.76, p<.01]$, reflecting fewer errors in the blank condition than in each of the other conditions $(p<.05)$. No other comparisons were significant.

A one-way ANOVA of the no-go RT data revealed an effect of condition $[F(3,69)=4.43, p<.01]$, reflecting significantly shorter RTs in the blank than in the face condition $(p<.05)$. Although RTs were also longest in the face condition, none of the other comparisons was significant. An ANOVA on the error data also revealed a main effect of condition $[F(3,69)=3.07]$, reflecting higher errors in the blank than in the name condition $(p<.05)$.

\section{Discussion}

As in Experiment 1, the famous faces delayed target RTs significantly more than did any of the comparison stimuli. These included a new class of nonface objects, images of national flags, and more important, names, which give the same status for persons as do the faces. In addition to Experiment 2, which eliminated face familiarity as an explanation for the findings of Experiment 1, we can now also exclude the personhood of visual stimuli as

Table 3

Mean Reaction Times (RTs, in Milliseconds), Standard Deviations ( $S D s)$, and Percentages of Errors $(\% \mathrm{E})$ as Functions of the Go and No-Go Conditions in Experiment 3

\begin{tabular}{cccccccc}
\hline & \multicolumn{3}{c}{ Go Condition } & & \multicolumn{3}{c}{ No-Go Condition } \\
\cline { 2 - 3 } \cline { 6 - 7 } Stimulus & RT & $S D$ & $\% \mathrm{E}$ & & RT & $S D$ & $\% \mathrm{E}$ \\
\hline Blank & 503 & 87 & 3 & & 557 & 112 & 18 \\
Object & 575 & 94 & 8 & & 602 & 106 & 11 \\
Name & 562 & 94 & 7 & & 611 & 120 & 11 \\
Face & 605 & 99 & 7 & & 652 & 206 & 14 \\
\hline
\end{tabular}


a likely explanation for these effects. Rather, an attention retention bias appears to reflect the status of faces per se. Nevertheless, it might be argued still that none of these experiments conclusively demonstrated an attention retention bias for faces. Alternatively, if faces initially capture attention more readily than do other stimuli under the present circumstances, this could produce a similar delay in target RTs on face trials. This was addressed in the next experiment.

\section{EXPERIMENT 4}

In our final experiment, we used a design identical to that in Experiment 1, except that we manipulated the stimulus onset asynchrony (SOA) between the central stimuli and the peripheral line targets. The purpose of this manipulation was to provide ample time for attention to be allocated equally to the face and the nonface stimuli, before the onset of the peripheral targets. Therefore, the face and nonface stimuli, and the superimposed go/no-go signals, were now presented $1,000 \mathrm{msec}$ prior to target onset. We reasoned that if a face bias is observed despite this relatively long SOA, this would provide further evidence for attention retention by faces.

\section{Method \\ Twenty-five new volunteer subjects were recruited. The stimuli and procedure were the same as those in Experiment 1, except for the following changes. Each trial began with a fixation dot for $750 \mathrm{msec}$, followed by a display consisting of the go/no-go signal superimposed on a face or a nonface distractor, which appeared for $1,000 \mathrm{msec}$. Following this, the peripheral line targets were added to the display for $200 \mathrm{msec}$, when a blank screen replaced all the stimuli until a response was registered. The subjects were requested to focus on the center of the display and, on go trials, to localize the line target (i.e., left or right of fixation). For no-go trials, the subjects were instructed to press the space key as soon as the target lines ap- peared, to initiate the next trial.}

\section{Results}

The median RTs and error rates, averaged across subjects, are shown in Table 4 as a function of the go and no-go conditions. For go trials, the RT data showed a main effect of condition $[F(3,72)=4.39, p<.01]$, with significantly slower responses in the upright face condition than in the blank, inverted face, and object conditions (Tukey HSD, $p<.05)$. However, unlike in the previous experiments, the blank condition did not differ from the inverted face and the object conditions. Analysis of the go error data did not show an effect of condition $[F(3,72)=0.16]$.

The no-go data were analyzed as were the go data. An ANOVA revealed no effects of condition for RTs $[F(3,72)=0.63]$ or error rates $[F(3,72)=2.33]$.

\section{Discussion}

With the same stimuli, this experiment replicated the most important aspects of Experiment 1. Thus, target RTs were delayed more by the presence of an upright face than by that of an inverted face or a meaningful nonface object. Importantly, this effect was now found despite a
Table 4

\begin{tabular}{|c|c|c|c|c|c|c|}
\hline \multirow[b]{2}{*}{ Stimulus } & \multicolumn{3}{|c|}{ Go Condition } & \multicolumn{3}{|c|}{ No-Go Condition } \\
\hline & RT & $S D$ & $\% \mathrm{E}$ & RT & $S D$ & $\% \mathrm{E}$ \\
\hline Blank & 377 & 59 & 4 & 307 & 117 & 5 \\
\hline Object & 378 & 73 & 4 & 319 & 138 & 11 \\
\hline Inverted face & 381 & 60 & 4 & 313 & 125 & 6 \\
\hline Upright face & 401 & 86 & 4 & 333 & 178 & 10 \\
\hline
\end{tabular}

1,000-msec SOA between the presentations of the faces and the line targets, thus providing considerable time for all the stimuli to engage processing resources prior to target onset. As another consequence of the SOA, RTs in the blank condition did not differ from those in the object and inverted face conditions in this task. This contrasts qualitatively with the pattern repeatedly found in Experiments 13 , where responses were always significantly faster in the blank condition than in all the other conditions. In fact, this advantage for the blank condition suggests that all the stimulus categories (upright and inverted faces, objects, and names) may have been able to capture or hold attention to some extent in these experiments. Because of the SOA manipulation, this effect appears to have been absent in Experiment 4, despite the overall presence of the same upright face bias as that in the previous experiments. As a result, Experiment 4 provides the strongest evidence yet that upright face stimuli are particularly efficient in retaining visual attention.

\section{GENERAL DISCUSSION}

Our study shows that the presentation of an upright face in the location of an attended go/no-go signal delays the classification of a peripheral target. Although target classification was delayed by the presence of any visual stimuli, relative to the blank condition in Experiments 1-3, upright faces always produced the largest delay. A variety of stimuli, including upside-down faces, pictures of objects, and names, were indistinguishable in this task. Moreover, this bias for upright faces was still observed in Experiment 4, in which the onset of the target stimuli was manipulated to control for attentional capture by the face stimuli. Thus, these findings imply that faces may be particularly efficient at retaining visual attention.

Overall, these results converge with existing research on the relationship between faces and attention, although previous studies have examined whether faces are particularly adept at drawing attention (e.g., Mack et al., 2002; Ro et al., 2001). It remains to be seen whether attentional capture and retention are influenced by the same mechanisms. Note also that although there is neuroscientific evidence that faces may be processed by a specialized system (see, e.g., De Renzi, 2000; Kanwisher, McDermott, $\&$ Chun, 1997), an attentional bias toward faces does not necessarily imply that they have their own dedicated attentional capacity. Indeed, attentional biases have now been 
observed for a variety of stimuli-for example, substancerelated pictorial cues in alcohol users and smokers (Jones, Jones, Smith, \& Copley, 2003; Waters, Shiffman, Bradley, \& Mogg, 2003). Finally, it remains unclear why faces might retain attention. One possibility is that an attention bias is directed at monitoring faces for dynamic social information, such as changes in eye gaze, facial speech, or emotional expression. Equally, a retention bias might facilitate the face processing involved in making familiarity decisions. Future studies need to address these issues to provide clues as to why faces hold attention.

\section{REFERENCES}

Amir, N., Elias, J., Klumpr, H., \& PrZeworski, A. (2003). Attentional bias to threat in social phobia: Facilitated processing of threat or difficulty disengaging attention from threat? Behaviour Research \& Therapy, 41, 1325-1335.

Bradley, B. P., Mogg, K., Falla, S. J., \& Hamilton, L. R. (1998). Attentional bias for threatening facial expressions in anxiety: Manipulation of stimulus duration. Cognition \& Emotion, 12, 737-753.

BRuce, V., \& Young, A. W. (1986). Understanding face recognition. British Journal of Psychology, 77, 305-327.

Burton, A. M., Bruce, V., \& Hancock, P. J. B. (1999). From pixels to people: A model of familiar face recognition. Cognitive Science, 23, 1-31.

Burton, A. M., \& Young, A. W. (1999). Simulation and explanation: Some harmony and some discord. Cognitive Neuropsychology, 16, 73-79.

De Renzi, E. (2000). Prosopagnosia. In M. J. Farah \& T. E. Feinberg (Eds.), Patient-based approaches to cognitive neuroscience (pp. 8596). Cambridge, MA: MIT Press.

Farah, M. J., Wilson, K. D., Drain, H. M., \& Tanaka, J. R. (1995) The inverted face inversion effect in prosopagnosia: Evidence for mandatory, face-specific perceptual mechanisms. Vision Research, 35, 2089-2093.

Fox, E., Russo, R., Bowles, R., \& Dutton, K. (2001). Do threatening stimuli draw or hold visual attention in subclinical anxiety? Journal of Experimental Psychology: General, 130, 681-700.

Jones, B. T., Jones, B. C., Smith, H., \& Copley, N. (2003). A flicker paradigm for inducing change blindness reveals alcohol and cannabis information processing biases in social users. Addiction, 98, 235-244.
Kanwisher, N., McDermott, J., \& Chun, M. M. (1997). The fusiform face area: A module in human extrastriate cortex specialized for face perception. Journal of Neuroscience, 17, 4302-4311.

LaVIE, N. (1995). Perceptual load as a necessary condition for selective attention. Journal of Experimental Psychology: Human Perception \& Performance, 21, 451-468.

Lavie, N. (2000). Selective attention and cognitive control: Dissociating attentional functions through different types of load. In S. Monsell \& J. Driver (Eds.), Control of cognitive processes: Attention and performance XVIII (pp. 175-194). Cambridge, MA: MIT Press, Bradford Books.

Mack, A., Pappas, Z., Silverman, M., \& Gay, R. (2002). What we see: Inattention and the capture of attention by meaning. Consciousness \& Cognition, 11, 488-506.

Moscovitch, M., Winocur, G., \& Behrmann, M. (1997). What is special about face recognition? Nineteen experiments on a person with visual object agnosia and dyslexia but normal face recognition. Journal of Cognitive Neuroscience, 9, 555-604.

Palermo, R., \& Rhodes, G. (2003). Change detection in the flicker paradigm: Do faces have an advantage? Visual Cognition, 10, 683-713.

Ro, T., Russell, C., \& Lavie, N. (2001). Changing faces: A detection advantage in the flicker paradigm. Psychological Science, 12, 94-99.

Shelley-Tremblay, J., \& Mack, A. (1999). Metacontrast masking and attention. Psychological Science, 10, 508-515.

Treisman, A. M. (1993). Representing visual objects. In D. E. Meyer \& S. Kornblum (Eds.), Attention and performance XIV: Synergies in experimental psychology, artificial intelligence, and cognitive neuroscience (pp. 163-175). Cambridge, MA: MIT Press.

VuILleumier, P. (2000). Faces call for attention: Evidence from patients with visual extinction. Neuropsychologia, 38, 693-700.

Waters, A. J., Shiffman, S., Bradley, B. P., \& MogG, K. (2003). Attentional shifts to smoking cues in smokers. Addiction, 98, 14091417.

Yiend, J., \& Mathews, A. (2001). Anxiety and attention to threatening pictures. Quarterly Journal of Experimental Psychology, 54A, 665-681.

Young, A. W., \& Burton, A. M. (1999). Simulating face recognition: Implications for modelling cognition. Cognitive Neuropsychology, 16, $1-48$.

(Manuscript received July 16, 2004; revision accepted for publication April 19, 2005.) 